Unfallchirurg 2011 · 114:192

DOI 10.1007/s00113-010-1891-8

Online publiziert: 11. März 2011

(c) Springer-Verlag 2011

\author{
T. Mittlmeier ${ }^{1}$. C. Krettek ${ }^{2}$ \\ ${ }^{1}$ Abteilung für Unfall- und Wiederherstellungschirurgie, \\ Universitätsklinik und Poliklinik für Chirurgie, Rostock \\ ${ }^{2}$ Unfallchirurgische Klinik, Medizinische Hochschule Hannover
}

\title{
Infektionen in der Unfallchirurgie
}

enthaltes eine MRSA-Infektion zu entwickeln, MRSA-Infektionen bedingen einen längeren stationären Aufenthalt und gehen mit einer höheren Mortalität einher. Staphylococcus-aureus ist mit knapp 40\% der häufigste Erreger von postoperativen Wundinfektionen in Orthopädie und Traumatologie, wobei postoperative Infektionen heute an dritter Stelle bei den nosokomialen Infektionen stehen.

\section{$>$ Seit Jahren sind schwere Strepto- und Staphylokokken-, aber auch Anaerobierinfek- tionen auf dem Vormarsch}

Auch unter ökonomischen Aspekten sind wir alle neben den bereits gültigen gesetzlichen Verpflichtungen aufgerufen adäquate Präventionsmaßnahmen wie Hygienestrategien und Surveillance- und Screeningprogramme zu nutzen, um den Krankenhausproblemkeimen Herr zu werden. Allein eine verbesserte Händehygiene ist eine effektive Maßnahme nosokomiale Infektionen um bis zu einem Drittel zu reduzieren. Die Kenntnisse chirurgischer Strategien zur Infektbekämpfung im Konzert mit weiteren Verfahren sind aus unserer Sicht für jeden operativ tätigen Unfallchirurgen und Orthopäden von Bedeutung, da eine verzögerte und das vorhandene Repertoire nicht ausschöpfende Therapie häufig schlechtere Resultate nach sich zieht. Die Behandlung des infizierten diabetischen Fußsyndroms mag als Beispiel dafür dienen, dass nur die interdisziplinäre Interaktion zwischen den operativ Tätigen, dem Angiologen, dem Diabetologen, dem interventionellen Radiologen und dem Mikrobiolo- gen entsprechend den gültigen Leitlinien reproduzierbar gute und Richtung weisende Resultate unter Extremitätenerhalt erbringen kann [2].

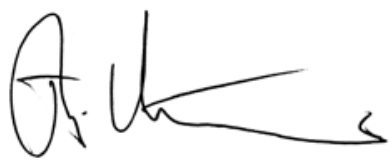

T. Mittlmeier

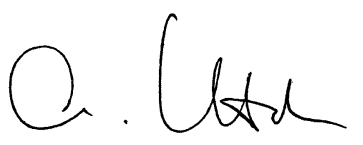

C. Krettek

\section{Korrespondenzadresse \\ Prof. Dr. T. MittImeier}

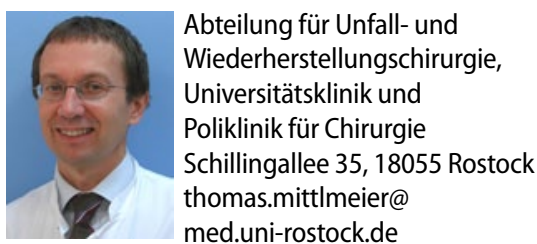

\section{Prof. Dr. C. Krettek}

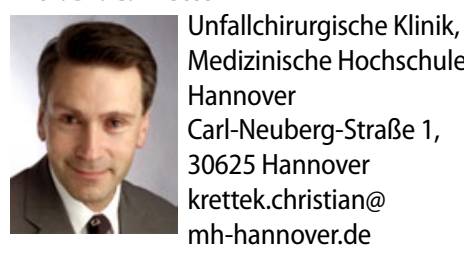

\section{Literatur}

1. Sarani, B, Strong M, Pascual J, Schwab CW (2009) Necrotizing fasciitis; current concepts and review of the literature. J Am Coll Surg 208:279-288

2. Weigelt JA (2010) Diabetic foot infections: diagnosis and management. Surg Infections 11:295-298 